\title{
Could the ABO Blood Group Types be Associated with COVID-19 Mortality?
}

\author{
๑ Sema Alacam, (1) Nagehan Didem Sari* \\ University of Health Sciences Turkey, Istanbul Training and Research Hospital, Clinic of Medical Microbiology, Istanbul, Turkey \\ *University of Health Sciences Turkey, Istanbul Training and Research Hospital, Clinic of Infectious Diseases and Clinical Microbiolog, Istanbul, Turkey
}

\section{Abstract}

Aim: Currently, it is still a matter of debate whether a particular blood types is associated with an increased risk of severe acute respiratory syndrome coronavirus 2 (SARS-CoV-2) infection. In the present study, we aimed to evaluate the relationship between the ABO blood group types and mortality in patients hospitalized with the diagnosis of Coronavirus disease-2019 (COVID-19).

Methods: This cross-sectional study included 427 patients diagnosed with COVID-19 between March 2020 and June 2020 . SARS-CoV-2 ribonucleic acid was investigated in the naso/oropharyngeal swab samples of the patients by polymerase chain reaction. The blood group types, clinical and demographic data of the cases were obtained from the hospital automation system and patient files.

Results: The age range of the patients was between 18 and 96, and 53.4\% (228/427) of them were male. The ABO blood group distribution was $47.1 \%$ A, 29.5\% O, 15.9\% B, 7.5\% AB. The overall mortality rate of COVID-19 cases was 16.9\%. COVID-19 mortality risks were not significant in any of the $\mathrm{ABO}$ blood group types.

Conclusion: We did not find a significant relationship between blood group types and mortality in patients who were hospitalized with a diagnosis of COVID-19 and received treatment and follow-up.

Keywords: Blood groups, SARS-CoV-2, COVID-19, mortality

\section{Introduction}

Coronavirus disease-2019 (COVID-19), caused by severe acute respiratory syndrome coronavirus 2 (SARSCoV-2), continues to spread worldwide with increasing morbidity and mortality rates (1). More than 149 million confirmed cases and approximately 3.5 million deaths were reported by the World Health Organization on May 23, 2021 (2). COVID-19 may exhibit a wide range of clinical manifestations including respiratory, cardiovascular, hematopoietic, gastrointestinal, neurological and immune systems (3). Considering the health, social and economic impacts of the COVID-19 outbreak, understanding the susceptibility to disease and clinical outcomes is critical (4). Current clinical observations have shown that age, gender, and comorbid diseases play a role an important risk factors in the pathogenesis and prognosis of COVID-19 (5). Hematological markers and radiological findings have been associated with a worse clinical outcome (6). Hyperfibrinogenemia, lymphopenia, D-dimer elevation and leukopenia were found to be significantly different between mild/moderate and severe COVID-19 patient groups (7).

Beginning with the pioneering study of Helmbold and Vogel (8) in the early 1960s, the ABO blood group polymorphism has been investigated among infectious disease risk and susceptibility factors for many pathogens. The $A B O$ blood group system basically includes $A$ and $B$ antigens and their corresponding antibodies. Differences in blood group, antigen expression can increase or decrease the susceptibility of the host to many infections. In addition, blood group antigens can alter the innate immune response to infection by facilitating intracellular uptake, signal transduction, or cell adhesion (9). Due to the variable clinical manifestations of SARS-CoV-2 infection and the severe impact of COVID-19 all over the world, intense interest in genetic and environmental factors has increased (10). During the COVID-19 pandemic, it has been hypothesized that the ABO blood group types may be associated with susceptibility to SARS-CoV-2 infection, and also that the effect of blood types on COVID-19

Address for Correspondence: Sema Alacam, University of Health Sciences Turkey, Istanbul Training and Research Hospital, Clinic of Medical Microbiology, Istanbul, Turkey Phone: +90 5326822017 E-mail: semalacam@hotmail.com ORCID: orcid.org/0000-0001-7957-2906 Received: 31.05.2021 Accepted: 20.06.2021 
severity is based on the different aggregation of receptors to SARS-CoV-2 on the host cell surface (11).

Whether a particular blood type is associated with an increased risk of SARS-CoV-2 infection remains controversial. Although studies have proven that age and comorbidities increase the risk of infection, the effect of blood types on COVID-19 severity and mortality have not yet been confirmed (12). In the present study, it was aimed to evaluate the relationship between $A B O$ and $R h$ blood group types and mortality in patients hospitalized with the diagnosis of COVID-19.

\section{Methods}

\section{Study Design and Patients}

Ethics Committee approval of this study was obtained from the Istanbul Training and Research Hospital Ethics Committee with no need for consent (date: 21.05.2021, approval number: 2828). In the present study, which was designed as a cross-sectional study, 427 COVID-19 cases with positive real-time reverse transcriptionpolymerase chain reaction (RT-PCR) test for SARS-CoV-2 were evaluated from the hospital automation system and patient files. The study includes all adult hospitalized COVID-19 cases between March 2020 and June 2020, whose $A B O$ blood groups were determined. The blood group types, clinical and demographic data of the cases were obtained from the hospital information system and patient files. Mortality was defined as death from all causes during hospitalization. Patients were younger than 18 years of age who were hospitalized with the diagnosis of COVID-19 but had a negative PCR test and were treated as outpatients were excluded from the study.

In vitro, the anti-A antibody found in individuals with blood type $\mathrm{B}$ or $\mathrm{O}$ appears to antagonize the interaction between SARS-CoV-1 and the angiotensin-converting enzyme 2 (ACE-2) receptor expressed by host target cells $(13,14)$. Therefore, in addition, it was thought that it would be appropriate to examine patients with $B$ and $O$ blood groups and patients with $A$ and $A B$ blood groups separately in two different groups.

\section{Collection, Storage and Transport of Clinical Samples}

Naso/oropharyngeal swab samples were collected with dacron swabs according to current CDC-COVID-19 guidelines. Swabs were immediately placed in sterile transport tubes containing 2-3 mL of VNAT ${ }^{\mathrm{TM}}$ (Bio-Speedy ${ }^{\circledR}$ COVID-19 Transfer Tube). SARS-CoV-2 RNA in swab samples of patients was performed using one of the RT-PCR diagnostic kits (Bio-Speedy ${ }^{\circledR}$ SARS-COV-2 double gene RTqPCR Kit) (Bioeksen, Istanbul, Turkey). Viral RNA extraction from the swap sent to the laboratory in a VNAT tube was performed according to the manufacturer's instructions. PCR mixes were prepared with the products obtained from the extraction and then amplification was performed on the CFX96 Touch System (Bio-Rad Laboratories, Inc, United States). The results were evaluated in line with the recommendations of the kit manufacturer.

\section{Blood Group Types}

The $A B O$ and Rh blood group types were determined using gel colon agglutination test systems (Across Gel ${ }^{\circledR}$ Gel Centrifugal Cards, Dia Pro Medical Products Inc., Istanbul, Turkey).

\section{Statistical Analysis}

Data were analyzed using SPSS 22 (IBM Corp.) package program. The compliance of the variables to normal distribution was examined using visual methods (histogram etc.) and the Kolmogorov-Smirnov test. Data are expressed as median (interquartile range). Differences between groups were compared using the non-parametric Mann-Whitney $U$ test for continuous variables, and Pearson's chi-square or Fisher's Exact tests for categorical variables. Correlation between the $A B O$ and Rh blood groups, age, gender, and comorbidities with mortality was analyzed by Spearman's rank correlation test. The risk prediction [Odds ratio: (OR)] for COVID-19 mortality in different $A B O$ blood groups was calculated with a 95\% confidence interval. This OR value was considered significant if the confidence intervals of the OR value did not include a value of 1 . Results with a p-value of less than 0.05 were considered statistically significant.

\section{Results}

A total of 427 patients with a diagnosis of COVID-19 who were followed up in the clinical and intensive care unit and whose blood types were known were included in the study. The ages of these patients were between 18 and 96 and $53.4 \%(228 / 427)$ of them were males. A high proportion (47.1\%) of COVID-19 cases consisted of those with A blood group. The distribution of blood groups of all cases is shown in Graphic 1. The overall mortality rate of COVID-19 cases was $16.9 \%$. Mortality rates of patients with the $O / B$ blood group and $A / A B$ blood group were compared and no significant difference was found. Comorbidity was detected in $60 \%$ of the study group. Comorbidities were hypertension (36.8\%), diabetes mellitus (32.8\%), asthma (12.2\%), coronary artery disease $(12.2 \%)$, malignancies (10.8\%) and chronic kidney diseases $(7.5 \%)$, respectively. Hypertension and diabetes mellitus were the most common two comorbidities in both $O / B$ and $A / A B$ blood group patients. There was no significant difference between different blood groups in terms of pneumonia $(p=0.87)$, length of hospital stay 


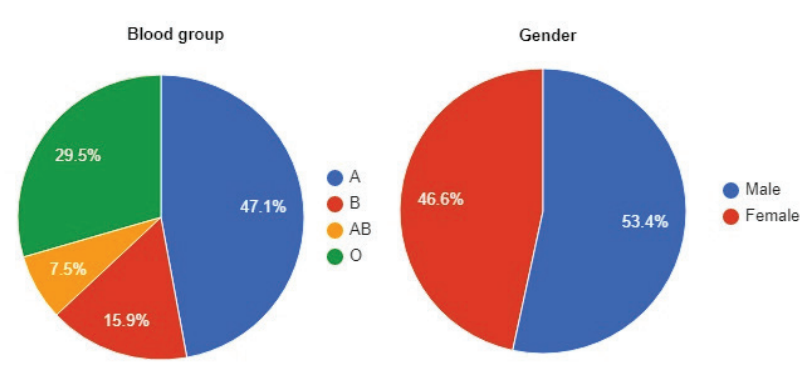

Graphic 1. Distribution of COVID-19 cases by blood group and gender

COVID-19: Coronavirus disease-COVID-19

$(p=0.79)$, and mortality rate $(p=0.94)$. The demographic characteristics, clinical and laboratory results of the cases are presented in Table 1.

The effects of risk factors such as age, gender and comorbidity on mortality were investigated in the patient population and no statistically significant difference was found. The relationship between blood group types, age, gender, and comorbidity with mortality in COVID-19 cases is presented in Table 2 . In addition, the relationship between age and mortality, independent of blood groups, was examined. The median age of patients who died was 60 years, and the median age of patients who recovered was 57 years, which was not statistically significant $(p=0.14)$.

COVID-19 mortality risk estimates (OR) were not statistically significant for any of the $A B O$ blood group types (Table 3).

\section{Discussion}

The effect of $A B O$ blood group types on COVID-19 disease severity is still being discussed. The relationship between blood group cells and SARS-CoV-2 can be attributed to some reasons. First, some coronaviruses can attach via surface proteins to extra sugar structures on the surface of A blood group cells that are missing in blood group 0 . Therefore, A blood group cells are likely to come into contact with more pathogens $(11,15)$. The other is that the relationship between the $\mathrm{S}$ protein of SARS-COV-2 and the ACE-2 protein on the host cell surface has been revealed. The binding of the spike protein to the ACE-2 receptor on the host cell surface, previously predicted for SARS-CoV, can be inhibited by the presence of an anti-A antibody $(15,16)$. It was stated that $A$ blood group can be a risk factor for hepatitis B virus (HBV) and human immunodeficiency virus (HIV), and group $\mathrm{O}$ can be protective for HBV, HCV, HIV, syphilis, or malaria (17). For these reasons, the effect of blood group types on COVID-19 clinical outcomes remains a focus of interest for

\begin{tabular}{|c|c|c|c|}
\hline Characteristics & O/B ( $n=194)$ & $A / A B(n=233)$ & $\mathbf{p}^{\star *}$ \\
\hline Male/female & $101 / 93$ & $127 / 106$ & 0.61 \\
\hline Rh positive $\mathrm{n}(\%)$ & $177(91.2)$ & $212(91)$ & 0.93 \\
\hline Age $^{*}$ & $55.5(40-68)$ & $59(49-70)$ & 0.02 \\
\hline Length of hospital stay* & $9(6-14)$ & $9(6-14)$ & 0.79 \\
\hline Mortality rate $\mathrm{n}(\%)$ & $33(17)$ & $39(16.7)$ & 0.94 \\
\hline Pneumonia n (\%) & $179(92.3)$ & $216(92.7)$ & 0.87 \\
\hline \multicolumn{4}{|l|}{ Comorbidity n (\%) } \\
\hline Hypertension & $68(35.1)$ & $89(38.2)$ & 0.50 \\
\hline Diabetes mellitus & $54(27.8)$ & $86(36.9)$ & 0.04 \\
\hline Asthma & $24(12.4)$ & $28(12)$ & 0.91 \\
\hline Chronic kidney disease & $8(4.1)$ & $24(10.3)$ & 0.02 \\
\hline Coronary artery disease & $25(12.9)$ & $27(11.6)$ & 0.68 \\
\hline Malignancy & $16(8.2)$ & $30(12.9)$ & 0.13 \\
\hline \multicolumn{4}{|c|}{ Laboratory parameter values* } \\
\hline Leukocyte $\left(\mathrm{x} 10^{3} / \mu \mathrm{L}\right)$ & $6.5(4.8-8)$ & $6.3(4.8-8.4)$ & 0.66 \\
\hline Neutrophil $\left(\times 10^{3} / \mu \mathrm{L}\right)$ & $4.2(3-5.8)$ & $4.1(3-6)$ & 0.99 \\
\hline 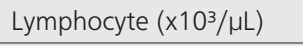 & $1.3(0.8-1.7)$ & $1.2(0.8-1.7)$ & 0.45 \\
\hline Hemoglobin $(\mathrm{g} / \mathrm{dL})$ & $13.3(12.1-14.5)$ & $13(11.3-14.2)$ & 0.03 \\
\hline Platelet $\left(\times 10^{3} / \mu \mathrm{L}\right)$ & $212(166-275)$ & $210(170-266)$ & 0.61 \\
\hline AST (U/L) & $30(21-47)$ & $30(24-40)$ & 0.92 \\
\hline $\operatorname{ALT}(\mathrm{U} / \mathrm{L})$ & $22(14-36)$ & $23(16-34)$ & 0.52 \\
\hline Creatine kinase (U/L) & $68(28-135)$ & $64(34-121)$ & 0.97 \\
\hline $\mathrm{LDH}(\mathrm{U} / \mathrm{L})$ & $250(183-348)$ & $244(178-329)$ & 0.50 \\
\hline Creatinine (mg/dL) & $0.8(0.6-1.1)$ & $0.9(0.7-1.1)$ & 0.48 \\
\hline CRP (mg /L) & $29.5(7.8-99.3)$ & $26(6.6-85.9)$ & 0.65 \\
\hline Procalcitonin (ng/mL) & $0.028(0-0.123)$ & $0.025(0-0.02)$ & 0.49 \\
\hline D-dimer $(\mathrm{ng} / \mathrm{mL})$ & $0.64(0.33-1.33)$ & $0.70(0.28-1.33)$ & 0.87 \\
\hline \multicolumn{4}{|c|}{$\begin{array}{l}{ }^{*} \text { Median (Interquartel range) }{ }^{* *} \text { Mann-Whitney } \mathrm{U} \text { test was used for median age, } \\
\text { Length of hospital stay and laboratory parameters and Pearson chi-square test } \\
\text { was used for other parameters. AST: Aspartate aminotransferase, ALT: Aspartate } \\
\text { alanine transaminase, LDH: Lactate dehydrogenase, CRP: C-reactive protein } \\
\text { COVID-19: Coronavirus disease-COVID-19 }\end{array}$} \\
\hline
\end{tabular}

researchers.

In this study, we investigated the effect of blood group types on mortality in 427 hospitalized patients with COVID-19. The overall mortality rate of COVID-19 cases was $16.9 \%$. We compared patients with blood groups $O$ and $B$ with anti-A antibodies and patients with blood groups $A$ and $A B$ without anti-A antibodies in terms of mortality risk with Spearman's correlation analysis and risk prediction analysis. We determined that there was no significant relationship between blood group types and mortality rate, mortality risk, and length of hospital stay in COVID-19 patients. In two different studies questioning the relationship between the $A B O$ group types and the risk of increased SARS-COV-2 infection and mortality, it was found that SARS-CoV-2 tends to infect individuals 


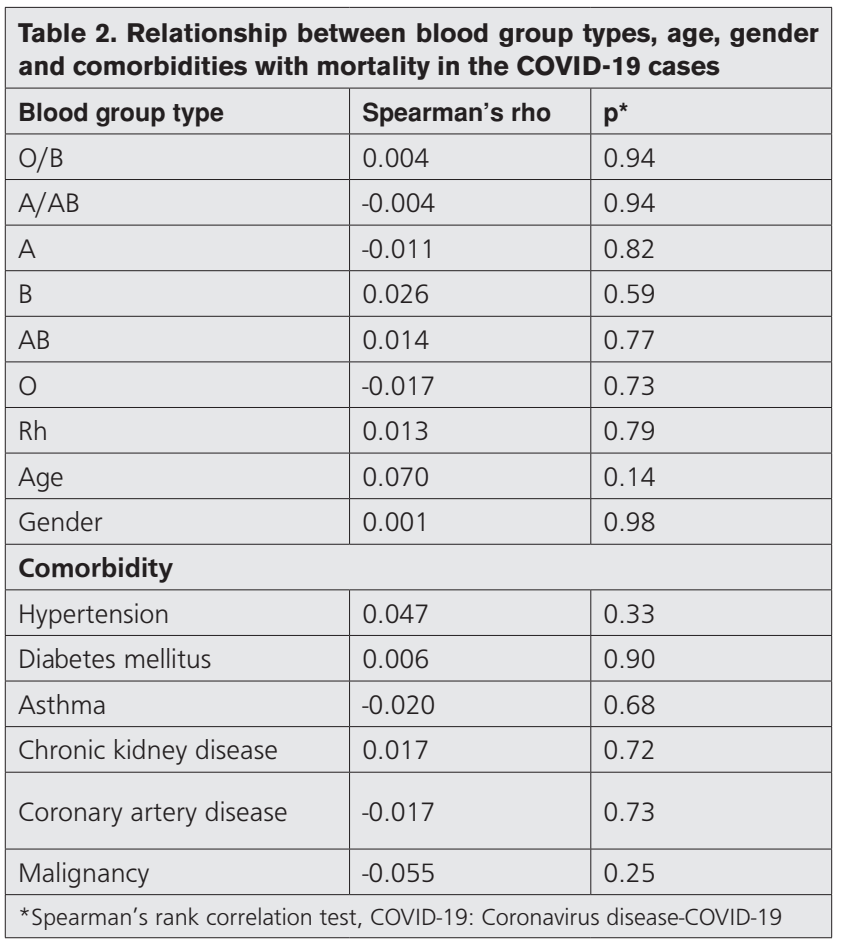

Table 3. Evaluation of mortality risk according to blood group types of COVID-19 cases

\begin{tabular}{|l|l|l|l|}
\hline Blood group types & Odds ratio & 95\% Confidence interval & $\mathbf{p}^{*}$ \\
\hline $\mathrm{A}$ & 0.9 & $0.5-1.5$ & 0.81 \\
\hline $\mathrm{B}$ & 1.2 & $0.6-2.3$ & 0.59 \\
\hline $\mathrm{AB}$ & 1.1 & $0.4-2.9$ & 0.76 \\
\hline $\mathrm{O}$ & 0.9 & $0.5-1.5$ & 0.72 \\
\hline $\mathrm{O} / \mathrm{B}$ & 1 & $0.6-1.6$ & 0.94 \\
\hline $\mathrm{A} / \mathrm{AB}$ & 0.9 & $0.5-1.6$ & 0.94 \\
\hline *Pearson's chi-square - odds ratio, COVID-19: Coronavirus disease-COVID-19 \\
\hline
\end{tabular}

with blood type $A$, while individuals with blood type $O$ have the lowest risk of infection $(18,19)$. Zhao et al. (19) reported that the rate of those with $A$ blood group was higher in deceased patients. Alkout and Alkout (20) found that individuals with $B$ and $O$ blood groups had milder COVID-19 disease. However, in subsequent studies, studies investigating the relationship between the $A B O$ blood group types and the risk of infection or disease outcome and containing conflicting results have been published $(3,21-23)$.

In some studies conducted in our country, it was reported that the risk of SARS-CoV-2 infection was lower in patients with O blood type (24). On the other hand, Solmaz and Araç (9) reported that blood groups did not affect the course of the disease and were not associated with mortality risk. In our study, the proportion of patients with blood group A who were hospitalized with the diagnosis of COVID-19 was higher. Many studies have shown that people with blood type A are significantly more susceptible to SARS-CoV-2 infection compared to blood type $O(18,19,25)$. There was no control group in this study. Therefore, it could not be investigated whether people with blood group A are more prone to this disease.

Genetic-based studies have also been conducted to investigate the effect of blood group types on COVID-19 mortality, and multiple genes and loci that may affect disease severity were found in the $A B O$ gene region $(26,27)$. However, in a recent study in the USA, it was determined that there was no significant difference between blood groups and mortality as a result of gene analysis of COVID-19 cases that died and survived (28). It is still controversial that blood group types may be a risk factor for COVID-19 mortality.

\section{Study Limitations}

This study does not include a control group. Therefore, susceptibility to SARS-CoV-2 infection could not be investigated among people with different blood groups. However, despite this limitation, it is one of the rare studies on this subject in Turkey. Due to the large patient population, the results of the study will contribute to the literature.

\section{Conclusion}

We did not find a significant relationship between blood group types and mortality in patients who were hospitalized with a diagnosis of COVID-19 and received treatment and follow-up. Although it has been reported that susceptibility to COVID-19 is decreased in individuals with blood type $O$, we think that prospective and comprehensive studies are needed to determine the role of blood group types on clinical outcomes and mortality in patients with COVID-19.

\section{Authorship Contributions}

Concept: S.A., N.D.S., Design: S.A., N.D.S., Data Collection or Processing: S.A., N.D.S., Analysis or Interpretation: S.A., N.D.S., Literature Search: S.A., N.D.S., Writing: S.A.

Conflict of Interest: No conflict of interest was declared by the authors.

Financial Disclosure: The authors declared that this study received no financial support.

\section{References}

1. Tuncer G, Surme S, Bayramlar OF, et al. NEWS2 and laboratory predictors correlated with clinical deterioration in hospitalised patients with COVID-19. medRxiv 20 January, 2021.

2. Information about COVID-19- World Health Organization Available at:https://covid19.who.int. Accessed 22 May, 2021. 
3. Dzik S, Eliason K, Morris EB, Kaufman RM, North CM. COVID-19 and ABO blood groups. Transfusion 2020;60:18834.

4. Niño M, Harris C, Drawve G, Fitzpatrick KM. Race and ethnicity, gender, and age on perceived threats and fear of COVID-19: Evidence from two national data sources. SSM Popul Health 2021;13:100717.

5. Zeng $X$, Fan $H$, Lu D, et al. Association between ABO blood groups and clinical outcome of coronavirus disease 2019: Evidence from two cohorts. Medrxiv April 17,2020.

6. Surme S, Buyukyazgan A, Bayramlar OF, et al. Predictors of Intensive Care Unit Admission or Death in Patients with Coronavirus Disease 2019 Pneumonia in Istanbul, Turkey. Jpn J Infect Dis. 2021 Feb 26.

7. Fu J, Kong J, Wang W, et al. The clinical implication of dynamic neutrophil to lymphocyte ratio and D-dimer in COVID-19: A retrospective study in Suzhou China. Thromb Res 2020;192:38.

8. Helmbold W, Vogel F. Correlations between ABO blood groups and epidemic diseases and their anthropological significance. Bibl Haematol 1962;13:279-80.

9. Solmaz I, Araç S. ABO blood groups in COVID-19 patients; Cross-sectional study. Int J Clin Pract 2021;75:e13927.

10. Anderson JL, May HT, Knight S, et al. Association of Sociodemographic Factors and Blood Group Type With Risk of COVID-19 in a US Population. JAMA Netw Open 2021;4:e217429.

11. Silva-Filho JC, Melo CGF, Oliveira JL. The influence of $A B O$ blood groups on COVID-19 susceptibility and severity: A molecular hypothesis based on carbohydrate-carbohydrate interactions. Med Hypotheses 2020;144:110155.

12. Göker H, Aladağ Karakulak E, Demiroğlu H, et al. The effects of blood group types on the risk of COVID-19 infection and its clinical outcome. Turk J Med Sci 2020;50:679-83.

13. Guillon $P$, Clément $M$, Sébille $V$, et al. Inhibition of the interaction between the SARS-CoV spike protein and its cellular receptor by anti-histo-blood group antibodies. Glycobiology 2008;18:1085-93.

14. Hoiland RL, Fergusson NA, Mitra AR, et al. The association of $A B O$ blood group with indices of disease severity and multiorgan dysfunction in COVID-19. Blood Adv 2020;4:49819.
15. Zaidi FZ, Zaidi ARZ, Abdullah SM, Zaidi SZA. COVID-19 and the $A B O$ blood group connection. Transfus Apher Sci 2020;59:102838.

16. Gérard C, Maggipinto G, Minon JM. COVID-19 and ABO blood group: another viewpoint. Br J Haematol 2020;190:934.

17. Batool Z, Durrani SH, Tariq S. Association Of Abo And Rh Blood Group Types To Hepatitis B, Hepatitis C, Hiv And Syphilis Infection, A Five Year' Experience In Healthy Blood Donors In A Tertiary Care Hospital. J Ayub Med Coll Abbottabad 2017;29:90-2.

18. Li J, Wang $X$, Chen J, Cai Y, Deng A, Yang M. Association between $\mathrm{ABO}$ blood groups and risk of SARS-CoV-2 pneumonia. Br J Haematol 2020;190:24-7.

19. Zhao J, Yang $Y$, Huang $H$, et al. Relationship between the $A B O$ Blood Group and the COVID-19 Susceptibility. Clin Infect Dis 2020:1150.

20. Alkout TA, Alkout AM. ABO blood groups among Coronavirus disease 2019 patients. Iberoamerican Journal of Medicine. 2020 Jun 13;2:268-74.

21. Leaf RK, Al-Samkari H, Brenner SK, Gupta S, Leaf DE. ABO phenotype and death in critically ill patients with COVID-19. Br J Haematol 2020;190:e204-e8.

22. Wu Y, Feng Z, Li P, Yu Q. Relationship between ABO blood group distribution and clinical characteristics in patients with COVID-19. Clin Chim Acta 2020;509:220-3.

23. Boudin L, Janvier F, Bylicki O, Dutasta F. ABO blood groups are not associated with risk of acquiring the SARS-CoV-2 infection in young adults. Haematologica 2020;105:2841-3.

24. Kirisci O, Ozluk S, Topalca US, Koprulu ND. Relationship of $\mathrm{ABO}$ Blood Groups to SARS-COV-2 Infection Causing COVID-19 Disease. Konuralp Medical Journal 2021;13:18-23.

25. Zietz M, Zucker J, Tatonetti NP. Associations between blood type and COVID-19 infection, intubation, and death. Nat Commun 2020;11:5761.

26. Ellinghaus D, Degenhardt F, Bujanda L, et al. Genomewide Association Study of Severe Covid-19 with Respiratory Failure. N Engl J Med 2020;383:1522-34.

27. Lehrer S, Rheinstein PH. ABO blood groups, COVID-19 infection and mortality. Blood Cells Mol Dis 2021;89:102571.

28. Kaiser J. Found: genes that sway the course of the coronavirus. Science 2020;370:275-6. 